\title{
Effect of ellagic acid on body fat and triglyceride reduction in healthy overweight volunteers: a randomized, double-blind, placebo-controlled parallel group study
}

\author{
Yoshiaki Shiojima $^{1}$, Megumi Takahashi ${ }^{1,2}$, Masayuki Kikuchi ${ }^{1,2}$ and Masanori Akanuma ${ }^{3}$
}

${ }^{1}$ Ryusendo Co., Ltd., Nishi-ikebukuro, Toshima-ku, Tokyo, Japan; ${ }^{2}$ Laboratory of Ultrasound Theranostics, Faculty of Pharma Sciences, Teikyo University, Japan; ${ }^{3}$ Akanuma Surgery Clinic, Ishiyama 2-jo, Minami-ku, Sapporo-shi, Hokkaido, Japan

Corresponding Author: Yoshiaki Shiojima, Ryusendo Co., Ltd., Nishi-ikebukuro, Toshima-ku, Tokyo, Japan

Submission Date: March 10 ${ }^{\text {th }}$, 2020, Acceptance Date: April $27^{\text {th }}, 2020$ Publication Date: April $30^{\text {th }}, 2020$

Citation: Shiojima Y., Takahashi M., Kikuchi M., and Akanuma M. Effect of ellagic acid on body fat and triglyceride reduction in healthy overweight volunteers: a randomized, double-blind, placebo-controlled parallel group study. Functional Foods in Health and Disease. 2020; 10(4): 180-194; DOI: https://doi.org/10.31989/ffhd.v10i4.702

\begin{abstract}
Background: Worldwide, those categorized as overweight or obese are increasing at an alarming rate, posing a serious public health problem. Current management methods vary, ranging from surgery, dieting and exercise, to the use of synthetic and natural compounds. Previous studies reported the use of an Irvingia gabonensis extract containing ellagic acid in reducing weight and other related parameters in overweight participants. The present study investigated the efficacy of ellagic acid on anthropometric parameters as well as body fat ratio and blood triglyceride levels in otherwise healthy overweight Japanese adults.
\end{abstract}

Participants and Methods: Overall, 32 participants (23 males and 9 females) aged between 20 and 64 years with a BMI of 25 or more but less than $30 \mathrm{~kg} / \mathrm{m}^{2}$ and a visceral fat area of $80 \mathrm{~cm}^{2}$ or more were included in this randomized double-blind clinical trial. The 20 -week intervention involved two groups of participants -placebo group and ellagic acid (3.0 mg per day) group. The placebo or ellagic acid was taken daily with water 30 minutes before the main meal. At baseline (T0) and at 6 and 12 weeks, anthropometric measurements (body weight, BMI, body fat ratio, waist circumference, hip circumference), CT scans and blood triglyceride levels were measured.

Results: Compared to the placebo, ellagic acid brought about statistically significant reductions in body fat ratio, triglycerides, body weight, BMI, waist circumference, hip circumference and visceral fat over the twelve-week trial period. 
Conclusion: The use of $3.0 \mathrm{mg}$ ellagic acid daily for a 12-week period was effective in reducing body fat ratio and blood triglycerides as well as other anthropometric parameters, confirming the potential use of ellagic acid in the management of overweight patients.

Keywords: Ellagic acid, Irvingia gabonensis, overweight, obesity, body fat, triglyceride, body weight, metabolic syndrome.

\section{BACKGROUND}

One out of three people in the world is obese or overweight [1]. The increasing prevalence of obesity is a public health issue that requires immediate attention. Globally, the rate of overweight people, i.e., those with body mass index (BMI) $\geq 25 \mathrm{~kg} / \mathrm{m}^{2}$, is increasing. In Western countries, $\mathrm{BMI} \geq 30 \mathrm{~kg} / \mathrm{m}^{2}$ is classified as obese. In 2015, there were approximately 2.2 billion overweight adults and children in the world, which accounted for approximately $30 \%$ of the world's population. Furthermore, the global population of obese children and adults, i.e., those with BMI $\geq 30 \mathrm{~kg} / \mathrm{m}^{2}$, were 17.7 million and 63.7 million, respectively [1]. The obesity rates have doubled since the 1980's in 70 countries and is steadily increasing in other countries and regions. Obesity is a multifaceted disease, and the accumulation of fat, particularly visceral fat, associated with obesity is linked with multiple complications such as type 2 diabetes mellitus, hypertension, dyslipidemia, metabolic syndrome, cerebral stroke, cancer, and an increase in overall morbidity and mortality $[2,3,4,5]$. In 2015, 4 million people died of diseases linked to obesity, which accounted for $7.1 \%$ of all cause of deaths.

According to the 2017 Japanese National Health and Nutrition Survey, 30.7\% of men and $21.9 \%$ of women aged 20 years and older were overweight (BMI $\geq 25 \mathrm{~kg} / \mathrm{m}^{2}$ ) and this rate increased to one in three men in their prime years; i.e., those aged 30-59 years, and one in four women aged 50 years and older [6]. Given these statistics, Japan introduced checkups for metabolic syndrome in 2008 for people aged 40-74 years. This was motivated by the knowledge that increased visceral fat causes metabolic syndrome, which increases the incidence of heart diseases such as myocardial infarction. Obesity or overweightness and accumulation of fat associated with excess body weight are caused by an imbalance in energy expenditure and intake; thus, optimizing the energy balance is essential for preventing excess weight or fat accumulation. According to the 2017 Japanese National Health and Nutrition survey, more than $80 \%$ of women with excess weight think about diet or exercise therapy but do not practice it, while only $13.2 \%$ of them practice it continuously. Thus, people who eat healthy diets and practice regular exercise are rare, even if they are aware of the importance of weight loss or prevention of metabolic syndrome.

Fat accumulation involves an increase in the size of fat cells (adipocyte hypertrophy) and an increase in the number of adipocytes (hyperplasia). Adipocytes do not only synthesize and accumulate fat, but also secrete adipocytokines, which play important roles in maintaining energy homeostasis, fat metabolism, immunity, and other physiological functions [7]. Therefore, modulating adipocyte function through foods and functional ingredients, and modulating adipocytokine secretion are expected to contribute to the prevention and improvement of obesity and type 2 diabetes mellitus. Polyphenols are some of the most well-known functional ingredients contained in foods. Various physiological functions of ellagic acid, a polyphenol contained in strawberries, pomegranates, walnuts, amla and African mango (Irvingia gabonensis) have been 
reported $[8,9,10,11]$. Ellagic acid has been part of traditional diets and used as a functional food ingredient worldwide with its action reported in many physiological and pharmacological studies $[12,13]$. Intake of food containing I. gabonensis-derived ellagic acid improved body weight, BMI, body fat ratio, triglycerides (TG), and waist circumference in overweight individuals [11]. Furthermore, intake of ellagic acid in I. gabonensis extract has been reported to be effective for body weight and body fat reduction. The main mechanism of action which involves the presence of triglycerides, is believed to be by downregulation of PPAR $\gamma$, a factor of adipocyte differentiation, to control adipogenesis, and this effect has been confirmed in a clinical trial [14, $15,16,17]$. There is however no data on the effectiveness of ellagic acid in Japanese people, studied in a randomized double-blinded placebo-controlled trial (RCT). Thus, this trial aimed to investigate the effect of ellagic acid on body fat reduction as determined by anthropometric measurements, blood triglyceride levels and abdominal computer tomography (CT) scans.

\section{METHODS}

A randomized double-blind placebo controlled parallel group study was carried out to determine the effect of ellagic acid on the anthropometry and blood triglyceride levels in overweight Japanese patients. The clinical trial protocol (Protocol No. 1901R) was approved by the Institutional Review Board of Akanuma Surgery Clinic (Hokkaido, Japan) in accordance with the ethical standards established in the Helsinki Declaration and the ethical guidelines for epidemiological and clinical research of the Ministry of Education, Culture, Sports, Science and Technology, and the Ministry of Health, Labor and Welfare of Japan. This study was registered in advance with the UMIN Clinical Trials Registry (http://www.umin.ac.jp) as UMIN000036773 and was conducted in compliance with the clinical trial protocol. Written informed consent was obtained from all participants. This study was conducted by a contract research organization, Kyowa Trial Co., Ltd. (Hokkaido, Japan) from May to October 2019 at the Akanuma Surgery Clinic (Hokkaido, Japan) and LSI Sapporo Clinic (Hokkaido, Japan).

\section{Participants}

Participants were recruited among individuals registered in a volunteer bank, accessed through the clinical trial division of Akanuma Surgery Clinic.

The inclusion criteria were as follows:

1. Males and females aged 20-64 years

2. Have a BMI of 25 or more but less than 30 (Level 1 Obesity by the Japan Society for the study of obesity: overweight by WHO criteria).

3. Have a visceral fat area of $80 \mathrm{~cm}^{2}$ or more

The exclusion criteria were as follows:

1. History of liver, kidney or heart disease, respiratory, endocrine, metabolic or neurological disorders, disturbance of consciousness, diabetes mellitus (according to the Japan Diabetes Society criteria), or other disease.

2. History of cardiovascular disease

3. Pharmacotherapy for sugar metabolism, fat metabolism, and hypertension 
4. Regular drug therapy or diet including health foods and supplements (including food for specified health uses, food with function claims, and food with nutrient function claims) that may interact with observations in this trial (except if the drugs, health foods, and supplements can be discontinued at the time of obtaining informed consent).

5. Allergies related to the investigational product

6. Previous experience of poor or worsened health after giving a blood sample

7. Blood donations $\geq 200 \mathrm{~mL}$ in the month before the trial or have plans for making a blood donation $\geq 200 \mathrm{~mL}$ during the trial period.

8. Participation in another clinical trial, whether ongoing or within the last four weeks

9. Heavy drinkers and smokers

10. Extremely irregular dietary patterns

11. Pregnant or breastfeeding women, women who might be pregnant, or are wishing to become pregnant during the trial period

12. Those who do not wish to provide their consent to the details of the trial explained to them in advance

Participants, who met the criteria and provided a written informed consent were assigned trial subject identification numbers (preliminary test numbers) for the screening test. The screening test assessed participants' age, medical history, whether they were being followed up at a hospital, medication, smoking, drinking, and exercising habits, allergies, and whether they used health foods and supplements. Participants underwent blood tests and their anthropometric parameters - height, body weight, BMI, body fat ratio, waist and hip circumferences, blood pressure, pulse and abdominal fat (total fat area- TFA, visceral fat area- VFA and subcutaneous fat area- SFA) were measured.

Figure 1 shows a flowchart from subject enrollment to analysis. The primary preliminary test was conducted on 121 individuals who volunteered and provided their consent for participation in the trial, and abdominal CT scan was taken as the secondary preliminary test on 81 individuals who met the selection criteria other than VFA. Forty-four individuals who met all the selection criteria were selected as subjects, and randomly assigned to either of two groups of 20, the placebo and ellagic acid groups. The latter would take an ellagic acid-containing capsule (3.0 mg/day). Twenty participants per group was the estimated enrollment for this study, and was based on a previous study on the effects of taking capsules containing I. gabonensis-derived ellagic acid (3 $\mathrm{mg} /$ day) for eight consecutive weeks on body weight and lipid parameters in overweight individuals (14 in the placebo group, 16 in the I. gabonensis-derived ellagic acid capsule group) [11]. The trial started with 44 subjects (22 in the placebo group and 22 in the ellagic acid group), who started taking the IP or placebo and comprised the full analysis set (FAS). The number of participants per group was to compensate for expected dropouts. The safety of the dose (ellagic acid $3.0 \mathrm{mg} /$ day) had been tested in previous clinical studies on ellagic acid contained in $I$. gabonensis extract, and in an unpublished study by the authors in which I. gabonensis extract capsules containing ellagic acid ( $15 \mathrm{mg} /$ day) were administered to 20 Japanese subjects for one month in a five-fold excessive dose trial [11, 14-17]. 


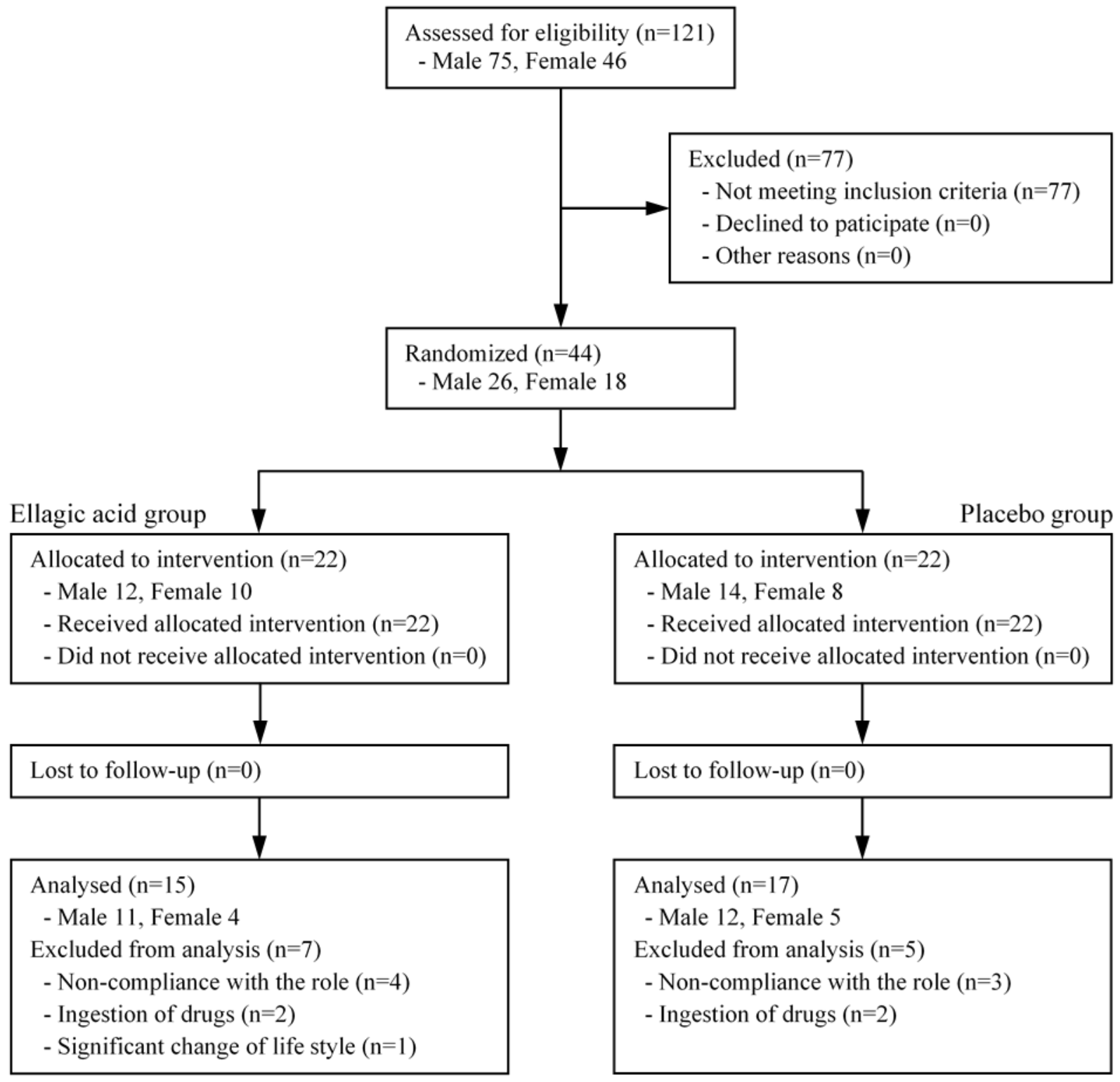

Figure 1. Flow diagram of the progress stages of this study

\section{Investigational product (IP)}

Ellagic acid powder used in this trial was prepared from I. gabonensis extract (IGOB131; Gateway Health Alliances, Inc., USA) with hot water, acid and alkaline, ethanol treatment, filtration, and then freeze-dried. The resulting powder ellagic acid was tested for purity by HPLC, using a Sigma standard. Ellagic acid was mixed with crystalline cellulose and silicon dioxide, and encapsulated in white capsules (hydroxypropyl methylcellulose; HPMC, titanium oxide) containing $1.5 \mathrm{mg}$ ellagic acid per capsule, and packaged in aluminum sachets (ellagic acid $3.0 \mathrm{mg} / 2$ capsules/sachet), which was equivalent to a single (daily) dose. The placebo was prepared by mixing crystalline cellulose and silicon dioxide, which were also encapsulated in white capsules (HPMC, titanium oxide), and packaged into two-capsule single daily-dose aluminum sachets, similar to the investigational product (IP), to ensure that they were identical in weight, appearance, and smell. Both study materials were prepared in a good manufacturing practice (GMP)-certified facility provided by Ryusendo Co., Ltd. (Tokyo, Japan). A dose of the IP was to be taken once a day with water or warm water $30 \mathrm{~min}$ before the main meal of the day. 


\section{Study design}

This trial was a randomized, double-blind, placebo-controlled parallel-group comparison study using an IP containing ellagic acid, and a placebo. The total trial period was 20 weeks, comprising 4 weeks of pre-administration observation, 12 weeks of administration, and 4 weeks of postadministration observation. A third party, with no relationship to the trial (Toyohira Koen Internal Medicine Clinic, Hokkaido, Japan), stratified the subjects by age and sex, then assigned them by stratified randomization using their VFA to ensure equal numbers of subjects in each group. During the four weeks of pre-administration observation and 12 weeks of administration of the IP (weeks 0,6 , and 12) and on week 4 of post-administration observation period, participants went for medical interview, anthropometric measurements, blood pressure, and blood tests.

The primary endpoints, body fat ratio, and TG were measured at the same intervals during administration of the IP; that is, at weeks 0,6 , and 12 , and on week 4 of the post-administration observation period. The secondary endpoints, body weight, BMI, waist and hip circumferences were also measured at the same intervals (weeks 0,6 , and 12) during IP administration and on week 4 of the post-administration observation period. The exploratory endpoints TFA, VFA, and SFA, were measured at baseline (before starting administration of the IP), and at week 12 of administration. Adverse events were investigated based on clinical test values, medical interviews, and records of physical activity kept by the participants.

Energy intake was not limited during the trial period and participants were instructed to maintain exercise levels as constant as possible. They submitted records of their diet and physical activity when they visited the hospital for tests taken at baseline (week 0) and at 6, and 12 weeks of taking the IP, and at week 4 of the post-administration observation. Participants were instructed to minimize intake of foods containing ellagic acid, including health foods and supplements, during the administration period, and to prohibit intake of foods, health foods, supplements, and drugs that may affect fat metabolism or body fat accumulation.

\section{Measurements of anthropometric and circulatory parameters}

The following anthropometric and circulatory parameters were measured: height (at the preliminary test only), body weight, BMI, body fat ratio, waist and hip circumference, body temperature, blood pressure, and pulse (measured twice with an automatic sphygmomanometer). Body mass index was calculated from the height and body weight.

\section{Measurement of abdominal fat areas}

TFA, VFA, and SFA were measured with CT scan following the methods described by Yoshizumi et al. [18]. The Aquilion ${ }^{\mathrm{TM}} 64$ CT System (Canon Medical Systems Co., Ltd., Japan) was used to take a body section in the transverse view at the level of the umbilicus, using the following x-ray parameters: D $120 \mathrm{kVp}, \mathrm{m}$ As value: "Auto" m As", window level 20 and window width 320. For image processing, a computer software for abdominal fat area analysis (Fat Scan ${ }^{\mathrm{TM}}$ Ver. 5.0; East Japan Institute of Technology Co., Ltd., Japan) was used to calculate TFA, VFA, and SFA. (*the $\mathrm{m}$ As value of "Auto' signifies that it was set at "Auto" rather than a fixed level "m" to adjust optimal doses for individual participants to minimize radiation exposure). 


\section{Measurement of blood triglyceride levels}

Fasting blood samples were taken at baseline (week 0) and at weeks 6 and 12 of administering the IP, and on week 4 of the post-administration observation period. Participants were prohibited from eating after 21:00 on the night prior to the blood sampling. They were however allowed to drink water. Blood triglyceride levels were measured using a biochemical kit (Pureauto S TG-N; Sekisui Medical Co., Ltd., Japan) and automatic analyzer (BioMajesty ${ }^{\mathrm{TM}}$ JCA-BM8040; JEOL Ltd., Japan).

\section{Record of diet and physical activity, and daily life diary}

Participants were encouraged not to modify their diets or lifestyle during the trial period. During the trial period, participants also recorded whether they took the IP, and details of their physical conditions, diet, and exercise in the diaries every day. Furthermore, they wore a Lifecorder GS calorie counter pedometer (Suzuken Co., Ltd., Japan) during the trial period to record their activity levels. Details on participants' health conditions were obtained through medical interviews that the chief investigator conducted on the various test days. Information on adverse events was also obtained by the chief investigator from the clinical test results, medical interviews, and participants' records of diet and physical activity.

\section{Statistical analysis}

Anthropometric values and changes $(\Delta)$ from baseline (week 0 ) are expressed as mean \pm standard error (SE). An unpaired t-test was used to test for significant intergroup differences between measured values at various intervals of taking the IP (weeks 0-12). An unpaired t-test was used to test for significant intergroup differences of changes $(\Delta)$ at various intervals of taking the IP (weeks 6 and 12). For intragroup comparison, paired t-test was used to find significant differences between various measurements at week 0 . The same test was used for comparison of abdominal fat area values measured at weeks 0 and 12 as well as for changes in measured values $(\Delta)$ from baseline (week 0). Actual values of blood triglyceride are expressed as mean \pm SE. Unpaired and paired tests were used for intergroup and intragroup comparisons, respectively, to find significant differences in the same manner as mentioned above. The Fisher's exact test was performed for comparison of adverse events. The statistical analysis software (SAS) version 9.4 (SAS Institute Inc., USA) was used for statistical analysis. For significance level, two-tailed $5 \%, 1 \%$, and $0.1 \%$, $<5 \%(\mathrm{P}<0.05),<1 \%(\mathrm{P}<0.01)$ and $<0.1 \%(\mathrm{P}<0.001)$ were expressed for significant difference. Correction for multiple comparison was not made for the paired t-test.

\section{RESULTS}

\section{Characterization of study group}

The trial started with 44 participants (22 in the placebo group and 22 in the ellagic acid group). Since there were no withdrawals or dropouts during the trial, all 44 participants completed the trial. The 44 eligible persons who started taking the IP comprised the full analysis set (FAS). Twelve participants who infringed on restrictions or met exclusion criteria were excluded. Thus, 32 were eligible for this protocol (placebo group, 17 subjects and ellagic acid group, 15 subjects), and comprised the per protocol set (PPS). The baseline characteristics of the PPS group are shown in Table 1. The mean age: placebo group was $52.2 \pm 6.6$ years while that of the ellagic acid group 
was $52.2 \pm 7.3$ years. The body weight for the placebo group was $74.7 \pm 6.5 \mathrm{~kg}$ and that of the ellagic acid group was $75.6 \pm 8.9 \mathrm{~kg}$, corresponding to a BMI of $27.2 \pm 1.5 \mathrm{~kg} / \mathrm{m}^{2}$ for the placebo group and $27.5 \pm 1.4 \mathrm{~kg} / \mathrm{m}^{2}$ for the ellagic acid group. The VFA for the placebo group was 146.3 $\pm 31.3 \mathrm{~cm}^{2}$, while that of the ellagic acid group was $157.1 \pm 37.9 \mathrm{~cm}^{2}$.

Table 1. Baseline characteristics of the subjects

\begin{tabular}{lcc}
\hline Parameter & Placebo group & Ellagic acid group \\
\hline $\mathrm{n}$ & 17 & 15 \\
Age (years) & $53.00 \pm 1.40$ & $49.87 \pm 1.75$ \\
Height $(\mathrm{cm})$ & $166.28 \pm 2.18$ & $168.11 \pm 2.45$ \\
Body weight $(\mathrm{kg})$ & $76.16 \pm 1.49$ & $77.05 \pm 1.97$ \\
Body mass index $\left(\mathrm{kg} / \mathrm{m}^{2}\right)$ & $27.58 \pm 0.38$ & $27.26 \pm 0.38$ \\
Body fat ratio $(\%)$ & $32.27 \pm 1.87$ & $31.46 \pm 1.61$ \\
\hline
\end{tabular}

Values represent the mean \pm SE. No significant differences were observed in any parameters between the two groups.

For the purposes of this manuscript, the safety assessment was based on the FAS. There were no significant intergroup differences in initial subject characteristics for any of the parameters.

Average intake rates of IP during the trial period in the placebo group and the ellagic acid group were $99.7 \pm 0.8 \%$ and $98.2 \pm 3.7 \%$, respectively, thus there was no difference between the groups.

An analysis of the records of diet (participants maintained habitual diets) and physical activity (steps taken) for six weeks during the trial period showed no intragroup changes or intergroup differences in any of the nutritional parameters, i.e., energy, protein, lipid, carbohydrate, and salt equivalent. There were also no significant intragroup changes and intergroup differences in terms of steps taken.

The changes from baseline in body weight, BMI, body fat ratio, waist and hip circumferences are shown in Table 2; changes in TG are shown in Table 3, and changes in abdominal fat area (TFA, VFA, and SFA) are shown in Table 4. There were no intergroup differences in any of the initial values.

After 12 weeks of IP administration, there were significant differences between the placebo and ellagic acid groups in the amount of change in body fat ratio, which is the primary endpoint (placebo group: $-0.18 \%$, ellagic acid group: $-1.37 \%(\mathrm{P}<0.01)$. In terms of actual measured values, there was a significant decrease from the original values (week 0) at weeks 6 and 12 in the ellagic acid group. Likewise, after 12 weeks of IP administration, there were significant differences between the placebo and ellagic acid groups in amount of change in TG, another primary endpoint (placebo group: $15.71 \mathrm{mg} / \mathrm{dL}$, ellagic acid group: $-22.80 \mathrm{mg} / \mathrm{dL}(\mathrm{P}<0.01)$ ). In terms of actual measured values, there was a significant decrease at week 12 compared to week 0 in the ellagic acid group. 
Table 2. Result of anthropometric measurements

\begin{tabular}{|c|c|c|c|c|c|}
\hline Parameter & Group & & $\begin{array}{l}0 \text { week } \\
\text { (Before) }\end{array}$ & 6 weeks & 12 weeks \\
\hline \multirow{4}{*}{$\begin{array}{l}\text { Body weight } \\
(\mathrm{kg})\end{array}$} & \multirow{2}{*}{ Placebo } & Measured value & $76.16 \pm 1.49$ & $75.69 \pm 1.47$ & $76.95 \pm 1.48$ \\
\hline & & Change value $\Delta$ & & $-0.46 \pm 0.27$ & $0.79 \pm 0.39$ \\
\hline & \multirow{2}{*}{ Ellagic acid } & Measured value & $77.05 \pm 1.97$ & $76.92 \pm 1.91$ & $75.99 \pm 1.86^{*}$ \\
\hline & & Change value $\Delta$ & & $-0.13 \pm 0.30$ & $-1.06 \pm 0.42^{\# \#}$ \\
\hline \multirow{4}{*}{$\begin{array}{l}\text { Body mass } \\
\text { index }\left(\mathrm{kg} / \mathrm{m}^{2}\right)\end{array}$} & \multirow{2}{*}{ Placebo } & Measured value & $27.58 \pm 0.38$ & $27.41 \pm 0.40$ & $27.87 \pm 0.42^{*}$ \\
\hline & & Change value $\Delta$ & & $-0.17 \pm 0.10$ & $0.29 \pm 0.14$ \\
\hline & \multirow{2}{*}{ Ellagic acid } & Measured value & $27.26 \pm 0.38$ & $27.23 \pm 0.39$ & $26.90 \pm 0.42^{*}$ \\
\hline & & Change value $\Delta$ & & $-0.03 \pm 0.10$ & $-0.36 \pm 0.14^{\# \#}$ \\
\hline \multirow{4}{*}{$\begin{array}{l}\text { Body fat ratio } \\
(\%)\end{array}$} & \multirow{2}{*}{ Placebo } & Measured value & $32.27 \pm 1.87$ & $31.55 \pm 1.97^{* *}$ & $32.09 \pm 1.95$ \\
\hline & & Change value $\Delta$ & & $-0.72 \pm 0.20$ & $-0.18 \pm 0.17$ \\
\hline & \multirow{2}{*}{ Ellagic acid } & Measured value & $31.46 \pm 1.61$ & $30.65 \pm 1.66^{* * * *}$ & $30.09 \pm 1.78^{* * *}$ \\
\hline & & Change value $\Delta$ & & $-0.81 \pm 0.18$ & $-1.37 \pm 0.29 \#$ \\
\hline \multirow{4}{*}{$\begin{array}{l}\text { Waist } \\
\text { circumference }\end{array}$} & \multirow{2}{*}{ Placebo } & Measured value & $95.45 \pm 1.16$ & $95.21 \pm 1.33$ & $97.22 \pm 1.44^{* *}$ \\
\hline & & Change value $\Delta$ & & $-0.25 \pm 0.31$ & $1.76 \pm 0.50$ \\
\hline & \multirow{2}{*}{ Ellagic acid } & Measured value & $94.49 \pm 1.58$ & $94.41 \pm 1.51$ & $93.81 \pm 1.58$ \\
\hline & & Change value $\Delta$ & & $-0.07 \pm 0.25$ & $-0.67 \pm 0.39^{\# \# \#}$ \\
\hline \multirow{4}{*}{$\begin{array}{l}\text { Hip } \\
\text { circumference } \\
(\mathrm{cm})\end{array}$} & \multirow{2}{*}{ Placebo } & Measured value & $102.62 \pm 1.00$ & $102.45 \pm 1.02$ & $103.59 \pm 1.09$ \\
\hline & & Change value $\Delta$ & & $-0.16 \pm 0.31$ & $0.97 \pm 0.48$ \\
\hline & \multirow[t]{2}{*}{ Ellagic acid } & Measured value & $100.13 \pm 1.20$ & $100.10 \pm 1.09$ & $99.30 \pm 1.16$ *\# \\
\hline & & Change value $\Delta$ & & $-0.03 \pm 0.27$ & $-0.83 \pm 0.33^{\# \#}$ \\
\hline
\end{tabular}

Values represent the mean $\pm S E$.

*, **, ***; $p<0.05,0.01,0.001$ vs. before $(0 w)$

\#, \#\#, \#\#; $p<0.05,0.01,0.001$ vs. placebo group 
Table 3. Effect of ellagic acid on triglycerides

\begin{tabular}{|c|c|c|c|c|c|}
\hline Parameter & Group & & $\begin{array}{c}0 \text { week } \\
\text { (Before) }\end{array}$ & 6 weeks & 12 weeks \\
\hline \multirow{4}{*}{$\begin{array}{l}\text { Triglycerides } \\
(\mathrm{mg} / \mathrm{dL})\end{array}$} & \multirow{2}{*}{ Placebo } & Measured value & $97.12 \pm 7.87$ & $122.41 \pm 15.38$ & $112.82 \pm 11.31$ \\
\hline & & Change value $\Delta$ & & $25.29 \pm 12.12$ & $15.71 \pm 8.84$ \\
\hline & \multirow{2}{*}{ Ellagic acid } & Measured value & $118.60 \pm 10.17$ & $123.80 \pm 15.08$ & $95.80 \pm 7.33^{*}$ \\
\hline & & Change value $\Delta$ & & $5.20 \pm 12.31$ & $-22.80 \pm 9.90$ \\
\hline
\end{tabular}

Values represent the mean $\pm S E$. *; $p<0.05$ vs. before $(0 w)$; \#\#; $p<0.01$ vs. placebo group

There were also significant differences between the placebo and ellagic acid groups in the magnitude of change in body weight and BMI after 12 weeks of IP administration. This was paralleled by a significant decrease at week 12 compared to week 0 in the ellagic acid group in terms of actual measured values as well. Similarly, a significant difference was observed between the placebo and ellagic acid groups in amount of change in waist circumference after 12 weeks of IP administration. In terms of actual measured values, a significant decrease was observed as well at weeks 6 and 12 compared to week 0 in the ellagic acid group. However, there was a significant increase on week 12 of taking the placebo. The placebo and ellagic acid groups differed significantly in amount of change in hip circumference after 12 weeks of IP administration, there was a significant intergroup difference at 12 weeks in terms of actual measured values, and a significant decrease at week 12 compared to week 0 in the ellagic acid group. No significant intergroup differences were observed in any of the other parameters.

Table 4. Effects of ellagic acid on total fat area, visceral fat area, and subcutaneous fat area

\begin{tabular}{|c|c|c|c|c|}
\hline Parameter & Group & & $\begin{array}{c}0 \text { week } \\
\text { (Before) }\end{array}$ & 12 weeks \\
\hline \multirow{4}{*}{$\begin{array}{l}\text { Total fat area } \\
\left(\mathrm{cm}^{2}\right)\end{array}$} & \multirow{2}{*}{ Placebo } & Measured value & $381.38 \pm 15.67$ & $377.65 \pm 16.90$ \\
\hline & & Change value $\Delta$ & & $-3.73 \pm 10.37$ \\
\hline & \multirow{2}{*}{ Ellagic acid } & Measured value & $397.14 \pm 15.87$ & $367.77 \pm 17.39^{* *}$ \\
\hline & & Change value $\Delta$ & & $-29.37 \pm 8.49$ \\
\hline \multirow{4}{*}{$\begin{array}{l}\text { Visceral fat area } \\
\left(\mathrm{cm}^{2}\right)\end{array}$} & \multirow{2}{*}{ Placebo } & Measured value & $145.98 \pm 7.18$ & $151.66 \pm 6.87$ \\
\hline & & Change value $\Delta$ & & $5.68 \pm 4.98$ \\
\hline & \multirow{2}{*}{ Ellagic acid } & Measured value & $157.97 \pm 10.91$ & $144.38 \pm 10.10^{* *}$ \\
\hline & & Change value $\Delta$ & & $-13.59 \pm 4.14^{\# \#}$ \\
\hline \multirow{4}{*}{$\begin{array}{l}\text { Subcutaneous fat } \\
\text { area }\left(\mathrm{cm}^{2}\right)\end{array}$} & \multirow{2}{*}{ Placebo } & Measured value & $235.39 \pm 15.73$ & $225.99 \pm 17.00$ \\
\hline & & Change value $\Delta$ & & $-9.40 \pm 7.24$ \\
\hline & \multirow[t]{2}{*}{ Ellagic acid } & Measured value & $239.15 \pm 13.27$ & $223.39 \pm 15.16^{*}$ \\
\hline & & Change value $\Delta$ & & $-15.76 \pm 5.84$ \\
\hline
\end{tabular}

Values represent the mean $\pm S E . *^{*}, * ; p<0.05,0.01$ vs. before $(0 w)$; \#\#; $p<0.01$ vs. placebo group

In amount of change in VFA, there was a significant difference between the placebo and ellagic acid groups after 12 weeks of IP administration (placebo group: $5.68 \mathrm{~cm}^{2}$, ellagic acid group: $-13.59 \mathrm{~cm}^{2}(\mathrm{P}<0.01)$ ). In terms of actual measured values, there was a significant decrease at week 12 compared to week 0 in the ellagic acid group. No significant intergroup differences 
were observed in TFA and SFA. However, they decreased significantly at week 12 compared to week 0 in the ellagic acid group.

During the study period, 12 cases for 7 subjects in the placebo group and 12 cases for 10 subjects in the ellagic acid group were reported as adverse events; however, none of these was a severe adverse event. Though the placebo group had significantly higher $(\mathrm{P}<0.05)$ levels of "headache" than the ellagic acid group, as reported in their records of daily life and medical interviews of the participants during the trial period. The chief investigator diagnosed them as headaches unrelated to the IP. There were no major abnormalities from the medical interviews by a physician, nor in subjective symptoms in the records or objective findings, and there were no concomitant symptoms.

\section{DISCUSSION}

A randomized double-blind placebo-controlled trial on otherwise healthy overweight individuals in Cameroon reported on the body fat and body weight reducing effects of food products containing I. gabonensis-derived ellagic acid [11]. However, it had not been tested whether these effects were caused by ellagic acid and if these effects could be extrapolated to the Japanese population. Thus, in the present trial, we investigated the effects of $3.0 \mathrm{mg}$ ellagic acid daily (before the main meal) for 12 consecutive weeks on body fat ratio, TG, body weight, BMI, waist and hip circumferences, and abdominal fat area (TFA, VFA, and SFA).

In the analysis of PPS $(n=32)$, there was a significant decrease in body fat ratio at week 6 ( $p$ $<0.001)$ and week 12 ( $\mathrm{p}<0.001)$ in the ellagic acid group; in contrast, it decreased at week 6 (p $<0.01$ ), but increase tendency was seen at week 12 in the placebo group. The amount of change observed between weeks 0 and 12 was $-0.18 \pm 0.17 \%$ for the placebo group and $-1.37 \pm 0.29 \%$ for the ellagic acid group. Thus, there was a significant body fat-decreasing effect after taking ellagic acid ( $\mathrm{p}<0.01)$. In contrast to the group taking ellagic acid, which decreased TG significantly at week 12 ( $\mathrm{p}<0.05)$, TG increased in the placebo group. The amount of change observed at week 12 compared to week 0 was $15.71 \mathrm{mg} / \mathrm{dL}$ for the placebo group and $-22.80 \mathrm{mg} / \mathrm{dL}$ for the ellagic acid group. Thus indicating a significant TG-decreasing effect of ellagic acid intake $(\mathrm{p}<0.01)$.

The changes observed at week 12 compared to week 0 in the various parameters were as follows: body weight (placebo group: $0.79 \pm 0.39 \mathrm{~kg}$ vs. ellagic acid group: $-1.06 \pm 0.42 \mathrm{~kg}, \mathrm{p}<$ 0.01 ), BMI (placebo group: $0.29 \pm 0.14 \mathrm{~kg} / \mathrm{m}^{2}$ vs. ellagic acid group: $-0.36 \pm 0.14 \mathrm{~kg} / \mathrm{m}^{2}, \mathrm{p}<0.01$ ), waist circumference (placebo group: $1.76 \pm 0.50 \mathrm{~cm}$ vs. ellagic acid group: $-0.67 \pm 0.39 \mathrm{~cm}, \mathrm{p}<$ 0.001 ), and hip circumference (placebo group: $0.97 \pm 0.48 \mathrm{~cm}$ vs. ellagic acid group: $-0.83 \pm 0.33$ $\mathrm{cm}, \mathrm{p}<0.01)$. Therefore, ellagic acid intake significantly decreased body weight, BMI, waist circumference, and hip circumference. The amount of changes in VFA at week 12 compared week 0 were $5.68 \pm 4.98 \mathrm{~cm}^{2}$ for the placebo group and $-13.59 \pm 4.14 \mathrm{~cm}^{2}$ for the ellagic acid group. Therefore, ellagic acid intake significantly decreased VFA $(\mathrm{p}<0.01)$. For TFA and SFA, there was no significant difference between the ellagic acid and placebo groups at week 12, but in the ellagic acid group, TFA and SFA decreased significantly at week 12, suggesting a marginal decrease in TFA and SFA by taking ellagic acid.

There were marginal but not significant increases in body fat ratio, TG, body weight, BMI, waist circumference, and VFA between weeks 0 and 12 in the placebo group. According to a study on seasonal changes in body composition in Japan, body weight and body fat ratio tend to decrease between March and July and tend to increase between July and November [19, 20]. Considering 
the trial period of this study (week 0: mid-May, week 6: late-June, week 12: mid-August), it was predicted that body weight and body fat ratio would somewhat decrease in the first half and increase in the second half. Indeed, body weight and fat decreased up to the midpoint (week 6) of the trial period in the placebo group but increased at week 12. The changes were similar in the ellagic acid group at week 6 . However, neither body weight nor body fat ratio increased at week 12; but significantly decreased at week 12 for the ellagic acid group ( $\mathrm{p}<0.05)$.

In the mechanisms underlying obesity, adipocytes differentiate and take in excess energy in the form of TG, and then expand to increase body fat. Thus, an increase in body weight is attributed to increase in body fat. A dose- and concentration-dependent steatogenesis-inhibiting effect of ellagic acid was shown in a trial of 3T3-L1 cells, which are cell line models for adipocytes [12]. Ellagic acid inhibited peroxisome proliferator-activated receptor $\gamma(\operatorname{PPAR} \gamma)$, the gene involved in regulating adipocyte differentiation, and inhibited the expression of $\mathrm{C} / \mathrm{EBP} \alpha$ and phosphorylation of retinoblastoma protein $[20,21,22]$. It is believed that when PPAR $\gamma$ activity is suppressed by these effects of ellagic acid, the TG content of adipose tissues decreases, thereby decreasing hypertrophy of adipocytes (obesity) [22]. Furthermore, in a trial of I. gabonensis extract (IGOB131) containing 1\% ellagic acid in the 3T3-L1 cell line, ellagic acid appeared to inhibit the activity of glycerol-3-phosphate dehydrogenase, an enzyme involved in transforming intracellular TG and glucose into TG to be stored in adipocytes [23]. Moreover, a decrease in leptin and an increase in adiponectin have also been confirmed. With the added effect of suppressing of the expression of PPAR $\gamma$ involved in adipocyte differentiation, ellagic acid appears to influence the transcription factor cascade, thereby inhibiting adipogenesis [24].

In the recent clinical study by Azantsa et al., taking I. gabonensis extract capsules containing ellagic acid ( $3 \mathrm{mg} /$ day) for 8 weeks decreased body weight, BMI, body fat ratio, waist and hip circumference, and TG significantly in overweight individuals $\left(26 \mathrm{~kg} / \mathrm{m}^{2} \leq \mathrm{BMI}<30 \mathrm{~kg} / \mathrm{m}^{2}\right)[11]$. It is inferred that the improvements observed in these parameters are attributable to ellagic acid. Similarly, from our results, lipid accumulation in adipose tissues and the liver is indeed associated with body weight gain and progression into becoming overweight. Changes in body weight are also partially attributed to the metabolic response and differentiation of adipose tissues. These mechanisms would explain the hypertrophy and hyperplasia of adipocytes related to overweightness and obesity. Furthermore, ellagic acid is a dietary compound that decreases fat, and its inhibitory action on adipogenesis appears at least partially associated with epigenetic modifications. Ellagic acid targets numerous mechanisms including free fatty acid (FFA) synthesis, TG esterification, and FFA oxidation to decrease lipid accumulation in the liver [22]. In another study, ellagic acid attenuated the formation of new adipocytes and biosynthesis of fatty acids (FA) in the adipose tissues but reduced the synthesis of TG and FA and increased FA oxidation in the liver [24]. These findings suggest that ellagic acid decreases lipids via unique mechanisms in adipose tissues and the liver to decrease the progression from becoming overweight to obesity and fatty liver in a synergistic manner.

There is one concern in comparing our trial to the clinical study of Azantsa et al. This trial assumes that ellagic acid alone affects the various parameters such as body weight, body fat ratio, and TG. Whereas the effects of components other than ellagic acid ( $3 \mathrm{mg} / \mathrm{day})$ in the I. gabonensis extract (IGOB131) $300 \mathrm{mg} /$ day cannot be excluded. Thus, the authors analyzed IGOB131 and found the following main nutritional components: water $(2.0-7.5 \%)$, protein $(0.4-0.7 \%)$, lipids (1.0-1.6\%), ash (1.5-2.2\%), carbohydrate (91.5-95.0\%, of which sugars and dietary fiber 
accounted for $89.0-93.5 \%$ and $2.2-5.5 \%$, respectively). The main constituents were ellagic acid (0.9-1.4\%), glucose (81.5-86.5\%, which comprised starch, maltotriose, maltotetraose, maltopentaose, maltose, free glucose, and others composed of the glucose molecule), free galactose (0.25-0.40), lactic acid (0.60-0.65\%), and citric acid (0.16-0.33\%), among other constituents. Qualitative and quantitative tests of functional components of vitamin $\mathrm{C}$ and organic acids etc. were also performed, but these were not detected. Mono- $O$-methyl ellagic acid, di- $O$ methyl ellagic acid, tri- $O$-methyl ellagic acid, and other ellagic acid analogs were also undetected. Moreover, the peak absorption spectrum of ellagic acid in IGOB131 by means of highperformance liquid chromatography was analyzed to confirm that there were no ellagic acid analogs. Normally, the seeds of I. gabonensis contain ellagic acid analogs; however, IGOB131 is manufactured by special methods such as supercritical extraction in producing the raw ingredient, therefore we assumed that functional ingredients other than ellagic acid were eliminated. In addition, we could assume that ellagic acid was the active agent involved in producing the results in the present study as well as the clinical study of Azantsa et al.

Taking $3.0 \mathrm{mg} /$ day of ellagic acid capsules for 12 consecutive weeks decreased the primary endpoints, body fat ratio and TG. In addition, the secondary endpoints, body weight, BMI, and waist and hip circumferences and VFA, one of the exploratory endpoints in this study were decreased. TFA and SFA also decreased marginally. Therefore, daily intake of $3.0 \mathrm{mg}$ ellagic acid by Japanese subjects decreased body fat ratio and various other parameters, which were the same outcomes reported by Azantsa at el.

Results of anthropometrics, clinical tests, and safety studies, including assessment of adverse events, during the trial period showed that the placebo group had significantly higher levels of "headache" than the ellagic acid group. However, given that this occurred only in the placebo group, and there was no change from baseline, it was a symptom that could happen commonly, and may not have any causal relationship with the IP. The chief investigator further deemed that there were no side effects nor other safety issues associated with the 12-week administration of ellagic acid. There were no intragroup changes nor changes in intergroup differences in terms of nutrition and exercise during the trial period compared to the three-day preliminary observation period before the trial (before arriving at the hospital). This suggested that nutritional intake or amount of exercise during the trial period had no effect on the results of the trial.

\section{CONCLUSION}

Capsules containing ellagic acid were administered to adult men and women aged 20-64 years, who were classified as obesity level $1\left(25 \mathrm{~kg} / \mathrm{m}^{2} \leq \mathrm{BMI}<30 \mathrm{~kg} / \mathrm{m}^{2}\right)$ and had VFA $\geq 80 \mathrm{~cm}^{2}$, to test the efficacy of ellagic acid-containing capsules for overweightness. This regime resulted in changes in primary endpoints- body fat ratio, TG, significant intragroup differences, changes in the secondary endpoints- body weight, BMI, hip circumference; waist circumference, significant intragroup differences and significant decreases in the exploratory endpoint VFA. Furthermore, there was a significant within group decrease in TFA and SFA. Various safety tests during the trial period confirmed that there were no side effects nor any other safety issues associated with taking the ellagic acid-containing capsules. 
Abbreviations: BMI, body mass index; TG, triglyceride; TFA, total fat area; VFA, visceral fat area; SFA, subcutaneous fat area; PPAR, peroxisome proliferator-activated receptors; G3PDH, glycerol-3-phosphate dehydrogenase; FFA, free fatty acid; IGOB131, extract of Irvingia gabonensis.

Competing interests: YS, MT, and MK are employees of Ryusendo Co., Ltd., the study sponsor. MA is the resident investigator. Ryusendo provided the research funds to Kyowa Trial Co., Ltd. MA received a research grant from Kyowa Trial, and have no conflicts of interest to declare. YS, MT and MK were not involved in the interpretation of results and did not influence the outcomes at any stage of the clinical trial. All authors have declared that they have no other conflict of interest.

Author's contributions: YS, MT, MK, and MA contributed to the conception and the design of the study, acquisition of data, or analysis and interpretation of data. YS, MT, and MK drafted the manuscript for important intellectual content. All authors approved the version of the manuscript to be submitted.

Acknowledgements: We would like to thank everyone who cooperated with this study, the participants, Akanuma Surgery Clinic, LSI Sapporo Clinic, Kyowa Trial Co., Ltd., and the staff of each medical institution. We thank our group members at Ryusendo Co., Ltd. for their technical support and valuable discussions.

\section{REFERENCES:}

1. GBD 2015 Obesity Collaborators, Ashkan Afshin, Mohammad H Forouzanfar, et al. Health effects of overweight and obesity in 195 countries over 25 years. N Engl J Med. 2017, 377(1):13-27.

2. Bray GA. Medical consequences of obesity. J Clin Endocrinol Metab. 2004, 89(6):25832589.

3. Eckel RH, Grundy SM, Zimmet PZ. The metabolic syndrome. Lancet. 2005, 365:14151428.

4. Kahn BB, Flier JS. Obesity and insulin resistance. J Clin Invest. 2000, 106:473-481.

5. Hasani-Ranjbar S, Jouyandeh Z, Abdollahi M. A systematic review of anti-obesity medicinal plants. J Diabetes Metab Disord 2013, 12:28.

6. Ministry of Health, Labour and Welfare in Japan. National health and nutrition survey. 2017, 102-109.

7. Yu YH, Ginsberg HN. Adipocyte signaling and lipid homeostasis sequelae of insulinresistant adipose tissue. Circ Res. 2005, 96:1042-1052.

8. Vattem DAA, Shetty K. Biological functionality of ellagic acid: a review. Journal of Food Biochemistry. 2005, 29:234-266.

9. Lei F, Xing DM, Xiang L, Zhao YN, Wang W. 2003, Pharmakokinetic study of ellagic acid in rat after oral administration of pomegranate leaf extract. J Chromatogr B. 2003, 796:189-194.

10. Yasukawa Z, Ozeki M, Okubo T, Kondo S. Effect of amla (Emblica officinalis Gaertn.) 
extract ingestion on recovery of body temperature and blood flow following cold stress A aandomized double_-blind placebo — controlled Study-. Jpn Pharmacol Ther. 2018, 46(2):227-35.

11. Azantsa B, Kuate D,Chakokam R, Paka G, Bartholomew B, Nash R. The effect of extracts of Irvingia gabonensis (IGOB131) and Dichrostachys glomerata (Dyglomera) on body weight and lipid parameters of healthy overweight participants. Funct Foods Health Dis. 2015, 5(6):200-208.

12. Wang L, Li L, Ran X, Long M, Zhang M, Tao Y, Luo X et al. Ellagic acid reduces adipogenesis through inhibition of differentiation-prevention of the induction of $\mathrm{Rb}$ phosphorylation in 3T3-L1 adipocytes. Evid Based Complement Alternat Med. 2013, 2013:287534.

13. Woo MS, Choi HS, Seo MJ, Jeon HJ, Lee BY. Ellagic acid suppresses lipid accumulation by suppressing early adipogenic events and cell cycle arrest. Phytotherapy Res. 2015, 29(3):398-406.

14. Onakpoya I, Davies L, Posadzki P, Ernst E. The efficacy of Irvingia gabonensis supplementation in the management of overweight and obesity: a systematic review of randomized controlled trials. J Diet Suppl. 2013, 10(1):29-38.

15. Ngondi JL, Oben JE, Minka SR. The effect of Irvingia gabonensis seeds on body weight and blood lipids of obese subjects in Cameroon. Lipids Health Dis. 2005, 4:12.

16. Oben JE, Ngondi JL, Momo CN, Agbor GA, Sobgui CSM. The use of a Cissus quadrangularis/Irvingia gabonensis combination in the management of weight loss: a double-blind placebo-controlled study. Lipids Health Dis. 2008, 7:12.

17. Ngondi JL, Etoundi1 BC, Nyangono1 CB, Mbofung CMF, Oben JE. IGOB131, a novel seed extract of the west african plant Irvingia gabonensis, significantly reduces body weight and improves metabolic parameters in overweight humans in a randomized double-blind placebo controlled investigation. Lipids Health Dis. 2009, 8:7.

18. Yoshizumi T, Nakamura T, Yamane M, Islam AH, Menju M, Yamasaki K, Arai T et al. Abdominal fat: standardized technique for measurement at CT. Radiology. 1999, 211(1):283-286.

19. Oka T, Kato G. Variations in body weight and body composition with special attention to body fat in humans. Kuroshio Science. 2012, 5(2):161-167.

20. Yamashita S, Imachi Y, Muto S. Sex and regional differences in seasonal variations of percent body fat among young Japanese students. Bulletin of Kurashiki Sakuyo University \& Sakuyo Junior College. 2005, 38(2):51-63.

21. Woo MS, Choi HS, Seo MJ, Jeon HJ, Lee BY. Ellagic acid suppresses lipid accumulation by suppressing early adipogenic events and cell cycle arrest. Phytother Res. 2015, 29:398406.

22. Wu D, Ma X, Tian W. Pomegranate husk extract, punicalagin and ellagic acid inhibit fatty acid synthase and adipogenesis of 3T3-L1 adipocyte. J Funct Food. 2013, 5(2):633-641.

23. Oben JE, Ngondi JL, Blum K. Inhibition of Irvingia gabonensis seed extract (OB131) on adipogenesis as mediated via down regulation of the PPARgamma and Leptin genes and up-regulation of the adiponectin gene. Lipids Health Dis. 2008, 7:44.

24. Okla M, Kang I, Kim DM, Gourineni V, Shay N, Gu L, Chung S. Ellagic acid modulates lipid accumulation in primary human adipocytes and human hepatoma Huh7 cells via discrete mechanisms. J Nutr Biochem. 2015, 26(1):82-90 